\title{
Grupo de Redes e Computação Aplicada (GRECA)
}

\author{
Roseclea Duarte Medina ${ }^{1}$, Giliane Bernardi ${ }^{1}$, Andreia Rosangela Kessler \\ Mühlbeier $^{1}$, Andreia Solange Boss ${ }^{1}$, Camila Cerezer Possobom ${ }^{1}$, Fabrício \\ Herpich $^{1}$, Felipe Becker Nunes ${ }^{1}$, Gleizer Bierhalz Voss ${ }^{1}$, Rafaela Ribeiro Jardim ${ }^{1}$, \\ Taciano Balardin de Oliveira ${ }^{1}$, Érico Marcelo Hoff do Amaral ${ }^{2}$, Sandra Dutra \\ Piovesan $^{2}$, Jaziel Souza Lôbo ${ }^{3}$ \\ ${ }^{1}$ Programa de Pós-Graduação em Informática - Universidade Federal de Santa Maria \\ (UFSM) \\ ${ }^{2}$ Curso de Engenharia da Computação - Universidade Federal do Pampa (UNIPAMPA) \\ ${ }^{3}$ Instituto Federal de Sergipe (IFS) \\ \{rosecle.medina, bgiliane, dehiamuhlbeier, andreia.bos, camila.ccpossobom, \\ fabricio.herpich, nunesfb, gleizer.voss, rafa.rjardim, tacianobalardin, \\ ericohoffamaral, sanpiovesan,jaziel.lobo\}@gmail.com
}

O Grupo de Redes e Computação Aplicada está vinculado à Universidade Federal de Santa Maria (UFSM), localizado na Avenida Roraima, $n^{\circ} 1000$ - Centro de Tecnologia - Sala 384 - Bairro Camobi - Santa Maria - RS - Brasil. Tem como coordenadora a professora Dr $^{\mathrm{a}}$ Roseclea Duarte Medina e possui linhas de pesquisa nas seguintes áreas: Computação Móvel, Computação Ubíqua e Pervasiva e Redes de Computadores. O GRECA é composto por oito pesquisadores e oito estudantes de diferentes instituições de ensino. O site oficial do grupo é $<$ www.ufsm.br/greca $>$.

O objetivo do GRECA é consolidar-se como um grupo de pesquisa multidisciplinar, atuando nas áreas de Redes de Computadores e Computação Aplicada à Educação. Nas pesquisas de Redes, os trabalhos têm foco em Gerência de Redes, Transmissão de Dados, desenvolvimento de aplicativos/sistemas móveis e cientes de contexto, bem como arquiteturas de redes necessárias para suportar diferentes ambientes em Cloud Computing (como AVAs, Laboratórios Virtuais, etc). A área de Computação Aplicada à Educação objetiva prover inovações que contribuam para o Ubiquitous Learning (u-Learning), atuando em pesquisa e desenvolvimento de ambientes educacionais como AVAS, Laboratórios Virtuais, Ambientes Imersivos e Serious Games.

Os projetos concluídos são: U-SEA; SEDECA; ROAD; DIP; Contexto de Expertise e Localização Influenciando a Gerência de Ti; TSADA; Mobile Service Desk e Miss Marple. Quanto aos projetos em andamento: UVLE ${ }^{\text {QoC }}$; Education Data Mining; SistEX e TCN ${ }^{5}$. 